\title{
Author Correction: The physics of spreading processes in multilayer networks
}

Manlio De Domenico, Clara Granell, Mason A. Porter and Alex Arenas

Correction to: Nature Physics 12, 901-906 https://doi.org/10.1038/nphys3865 (2016), published online 22 August 2016.

In the version of this Progress Article originally published, the left and right panels of Fig. 3, clarifying the details indicated within the centre panel, were mistakenly interchanged. This has now been corrected in all versions of the Progress Article.

Published online: 23 February 2018

https://doi.org/10.1038/s41567-018-0065-4

\section{Publisher Correction: A quantum dipolar spin liquid}

N. Y. Yao iD, M. P. Zaletel, D. M. Stamper-Kurn and A. Vishwanath

Correction to: Nature Physics https://doi.org/10.1038/s41567-017-0030-7, published online 5 February 2018.

In the version of this Article originally published, the title for reference 11 was incorrect, and should have read 'Influence of the range of interactions in thin magnetic structures. This has been corrected in all versions of the Article.

Published online: 21 March 2018

https://doi.org/10.1038/s41567-018-0095-y

\section{Publisher Correction: Universality of clone dynamics during tissue development}

Steffen Rulands (D), Fabienne Lescroart, Samira Chabab, Christopher J. Hindley (D), Nicole Prior, Magdalena K. Sznurkowska, Meritxell Huch, Anna Philpott, Cedric Blanpain and Benjamin D. Simons (D)

Correction to: Nature Physics https://doi.org/10.1038/s41567-018-0055-6, published online 26 February 2018.

In the version of this Letter originally published, Steffen Rulands was not listed as a corresponding author. This has been corrected in all versions of the Letter.

Published online: 16 March 2018

https://doi.org/10.1038/s41567-018-0096-x 